\title{
Estimating Right Atrial Pressure Using Ultrasounds: An Old Issue Revisited With New Methods
}

\author{
Renato De Vecchis ${ }^{\mathrm{a}, \mathrm{e}}$, Cesare Baldi ${ }^{\mathrm{b}}$, Giuseppe Giandomenico ${ }^{\mathrm{c}}$, Marco Di Maio ${ }^{\mathrm{d}}$, Anna Giasi ${ }^{\mathrm{a}}$, \\ Carmela Cioppa $^{\mathrm{a}}$
}

\begin{abstract}
Knowledge of the right atrial pressure (RAP) values is critical to ascertain the existence of a state of hemodynamic congestion, irrespective of the possible presence of signs and symptoms of clinical congestion and cardiac overload that can be lacking in some conditions of concealed or clinically misleading cardiac decompensation. In addition, a more reliable estimate of RAP would make it possible to determine more accurately also the systolic pulmonary arterial pressure with the only echocardiographic methods. The authors briefly illustrate some of the criteria that have been implemented to obtain a non-invasive RAP estimate, some of which have been approved by current guidelines and others are still awaiting official endorsement from the Scientific Societies of Cardiology. There is a representation of the sometimes opposing views of researchers who have studied the problem, and the prospects for development of new diagnostic criteria are outlined, in particular those derived from the matched use of twoand three-dimensional echocardiographic parameters.
\end{abstract}

Keywords: Right atrial pressure; Ultrasound monitoring; Inferior vena cava; $3 \mathrm{D}$ echocardiography

\section{Introduction}

Non-invasive techniques for determination of right atrial pressure (RAP) are still the subject of active research and debate and hence there is value in a review which highlights the un-

Manuscript accepted for publication June 20, 2016

aCardiology Unit, Presidio Sanitario Intermedio "Elena d'Aosta", ASL Napoli 1 Centro, Napoli, Italy

bHeart Department, Interventional Cardiology, A.O.U. "San Giovanni di Dio e Ruggi d'Aragona", Salerno, Italy

'Hospital Directorate, Presidio Sanitario Intermedio "Elena d'Aosta", ASL Napoli 1 Centro, Napoli, Italy

dDepartment of Cardiology, Second University of Napoli, Monaldi Hospital, Napoli, Italy

${ }^{e}$ Corresponding Author: Renato De Vecchis, Cardiology Unit, Presidio Sanitario Intermedio "Elena d'Aosta", via Cagnazzi 29, c.a.p.80137, ASL Napoli 1 Centro, Napoli, Italy. Email: devecchis.erre@virgilio.it

doi: http://dx.doi.org/10.14740/jocmr2617w certainties and shortcomings of current knowledge. This subject has been widely debated for decades [1-4]; whilst there is a high degree of consensus on some key points, there are also clear differences of opinion on others. A first point of substantial agreement is the consideration that right heart catheterization is the gold standard method of measuring RAP [5]. Using this method, measurement is taken via a thermodilution catheter during hemodynamic evaluation of sections of the right heart. Other measurements taken usually include systolic pulmonary artery pressure (SPAP), mean pulmonary arterial pressure (mPAP; calculated as the diastolic pulmonary arterial pressure plus one-third of the pulmonary pulse pressure), pulmonary arteriolar resistance and pulmonary capillary wedge pressure (PCWP). PCWP is a good proxy for the end-diastolic left ventricular pressure [6]. Table 1 gives a list of normal pressure and resistance values obtained using right heart catheterization (continuous thermodilution technique).

The introduction of the Swan-Ganz floating balloon catheter, which is still in vogue, was a major step forward in the invasive evaluation of right cardiac pressures [7]. Such measurements are routinely taken in patients with cardiomyopathy or chronic ischemic heart disease and candidates for heart transplantation $[8,9]$. Knowledge of RAP as well as pulmonary vascular resistance is very important because it enables the physician to identify different profiles of hemodynamic severity of heart disease, making it possible to predict in advance the clinical and hemodynamic demeanor of the graft after placement in the receiver's thorax. If, for example, the receiver has relatively high pulmonary vascular resistances, the clinical course after transplantation is expected to be more complicated and/or challenging, as the right ventricle of the graft will be obliged to work against a high afterload $[10,11]$.

Even in relatively common conditions such as acute decompensated heart failure (ADHF), a precise hemodynamic profile of right-sided heart sections (RAP, SPAP, total pulmonary vascular resistance, in addition to the PCWP) would be valuable, not so much for diagnosis, which can be based on a thorough clinical observation, but because it offers the opportunity to optimize the dose of drugs used in the unloading therapy, i.e., intravenous diuretics and/or morphine and/ or intravenous vasodilators [12]. In particular, in cases of biventricular failure, after intravenous diuretic therapy has been already started, the knowledge of a well-definite value of RAP is very important, in order to enable the physician to reliably determine the degree of residual patient's hemodynamic con- 
Table 1. Hemodynamic Monitoring With a Pulmonary Artery Catheter: Normal Pressures and Resistance Values

\begin{tabular}{lll} 
& Mean & Range \\
\hline Right atrium & $4 \mathrm{~mm} \mathrm{Hg}$ & $1-8 \mathrm{~mm} \mathrm{Hg}$ \\
Right ventricle & & \\
Peak-systolic & $25 \mathrm{~mm} \mathrm{Hg}$ & $15-30 \mathrm{~mm} \mathrm{Hg}$ \\
End-diastolic & $9 \mathrm{~mm} \mathrm{Hg}$ & $4-12 \mathrm{~mm} \mathrm{Hg}$ \\
Pulmonary capillary wedge pressure & $9 \mathrm{~mm} \mathrm{Hg}$ & $4-12 \mathrm{~mm} \mathrm{Hg}$ \\
Systemic vascular resistance & 1,100 dyne-s $/ \mathrm{cm}^{5}$ & $700-1,600$ dyne-s $/ \mathrm{cm}^{5}$ \\
Pulmonary vascular resistance & 70 dyne-s/cm & $20-130$ dyne-s $/ \mathrm{cm}^{5}$ \\
\hline
\end{tabular}

gestion. This allows the physician to adopt with complete safety the decision to stop the intravenous administration of loop diuretics if not only clinical but also hemodynamic indicators of congestion have been adequately improved by therapy [13]. The adoption of such a decision in a patient who is recovering from recent (a few days before) episode of ADHF actually would indicate that the state of hemodynamic congestion has been resolved and that it is no longer necessary to continue the previously undertaken intensive unloading therapy. The decision to discontinue diuretics and/or vasodilators can be taken simply on the basis of the clinical picture, but several studies have shown that adjusting diuretic dosing based solely on clinical criteria is rather unsafe and carries a substantial risk of therapeutic error, in particular underestimation of the potential risks of subsequent short-term relapses of cardiac decompensation [14]. The risk of premature discontinuation of intravenous diuretic therapy in patients who appear to have normalized but are still affected by subclinical hemodynamic congestion can be minimized if the RAP value is available.

In patients with heart failure, especially in those who are frail or elderly, invasive monitoring by pulmonary artery catheterization (PAC) is a challenging and traumatic practice, entailing the risk of infectious complications at the venous access site in cases where the central venous catheter is in place for a prolonged period. However, hemodynamic optimization with PAC did not improve renal function or long-term outcomes in patients with ADHF [15]. There have been numerous attempts to find a non-invasive replacement, such as transthoracic echocardiography, for right heart catheterization. A number of noninvasive ultrasonographic methods for estimating RAP have been proposed and implemented. The purpose of this brief review is to present an overview of the various proposed noninvasive methods of estimating RAP and illustrate the role that they currently play in the management of heart failure.

\section{Techniques for Estimating RAP Based on Mor- phological and Functional Parameters of the Ostium and Distal Segment of the Inferior Vena Cava (IVC)}

The IVC carries venous blood from the lower body to the right atrium, into which it opens via an orifice, whose diam- eter varies considerably as a function of the diaphragmatic movements related to breathing. The respiro-phasic variations of the IVC ostium are dependent on changes in endothoracic pressure, which are influenced by the movements of the diaphragm that delimit the lower chest. During inspiration, the diaphragm moves down, causing an increase in endothoracic negative pressure and thus increasing the capacity of the pulmonary veins and causing an increase in venous return to the right ventricle and pulmonary circulation. The epiphenomenon is an increase in the speed of the centripetal venous flow through the caval vessels and a reduction in their diameter; also the pressure in the right atrium decreases. In contrast, during expiration, the blood return to the pulmonary circulation is reduced and, when the expiratory act is prolonged, as in the Valsalva maneuver, the RAP increases, as does the diameter of the IVC ostium. It is relatively easy to follow and measure the respiratory fluctuations in IVC ostium diameter using 2D Doppler echocardiography $[5,16-18]$. Reports usually refer to the maximum diameter of the IVC ostium, i.e., the value recorded at the end of a prolonged expiratory act (sniff). A maximum IVC diameter $\geq 21 \mathrm{~mm}$ is considered pathological, i.e., indicative of RAP $>8 \mathrm{~mm} \mathrm{Hg}$ [19].

\section{Current American Society of Echocardiography (ASE) Guidelines for the Non-Invasive Diagno- sis of High RAP}

In the context of chronic heart failure, a high ( $\geq 21 \mathrm{~mm})$ maximum IVC diameter indicates hemodynamic congestion; the greater the hemodynamic congestion, the greater the IVC ostial dilation. This criterion is endorsed in the ASE guidelines [19]. In these guidelines that have been left unchanged in the recent update [20], a diagnostic detection algorithm for high ( $>8 \mathrm{~mm} \mathrm{Hg}$ ) RAP is proposed (Table 2), which takes into account not only the maximum IVC diameter, but also the degree of inspiratory collapse of the distal tract of the IVC, i.e., the IVC collapsibility index (IVCCI) (Fig. 1). IVCCI is calculated using the following formula: (maximum IVC diameter - minimum IVC diameter/maximum IVC diameter $) \times 100$. According to the interpretation provided by Rudski et al in the current ASE guidelines [19], the discriminating value to be taken into account for IVCCI is $50 \%$. IVCCI values of $\geq 50 \%$ are as- 
Table 2. Estimation of RA Pressure on the Basis of IVC Diameter and Collapse According to Rudski et al [19]

\begin{tabular}{|c|c|c|c|}
\hline Variables & Normal (0 - 5(3) mm Hg) & Intermediate (5 - 10(8) $\mathrm{mm} \mathrm{Hg})$ & High (15 mm Hg) \\
\hline IVC diameter & $\leq 21 \mathrm{~mm}$ & $\leq 21 \mathrm{~mm} ;>21 \mathrm{~mm}$ & $>21 \mathrm{~mm}$ \\
\hline Collapse with sniff & $>50 \%$ & $<50 \% ;>50 \%$ & $<50 \%$ \\
\hline $\begin{array}{l}\text { Secondary indices } \\
\text { of elevated RAP }\end{array}$ & & & $\begin{array}{l}\text { Restrictive filling } \\
\text { Tricuspid E/e' }>6 \\
\text { Diastolic flow predominance } \\
\text { in hepatic veins (systolic } \\
\text { filling fraction }<55 \% \text { ) }\end{array}$ \\
\hline
\end{tabular}

Ranges are provided for low and intermediate categories, but for simplicity, midrange values of $3 \mathrm{~mm} \mathrm{Hg}$ for normal and $8 \mathrm{~mm} \mathrm{Hg}$ for intermediate are suggested. Intermediate $(8 \mathrm{~mm} \mathrm{Hg})$ RAPs may be downgraded to normal $(3 \mathrm{~mm} \mathrm{Hg})$ if no secondary indices of elevated RAP are present, upgraded to high if minimal collapse with sniff $(<35 \%)$ and secondary indices of elevated RAP are present, or left at $8 \mathrm{~mm} \mathrm{Hg}$ if uncertain. IVC: inferior vena cava; RAP: right atrial pressure. The table synthetically displays the concepts expressed by Rudski et al [19] in the official recommendations of the American Society of Echocardiography (2010). These criteria have been left unchanged in the recent update [20] (January 2015).

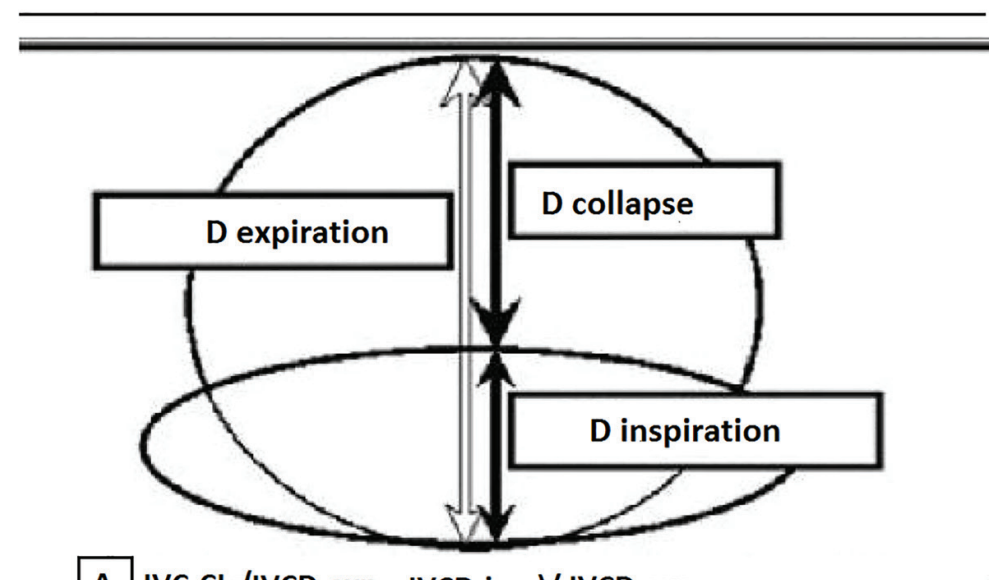

A IVC-Cl=(IVCD-exp - IVCD-insp) / IVCD-exp

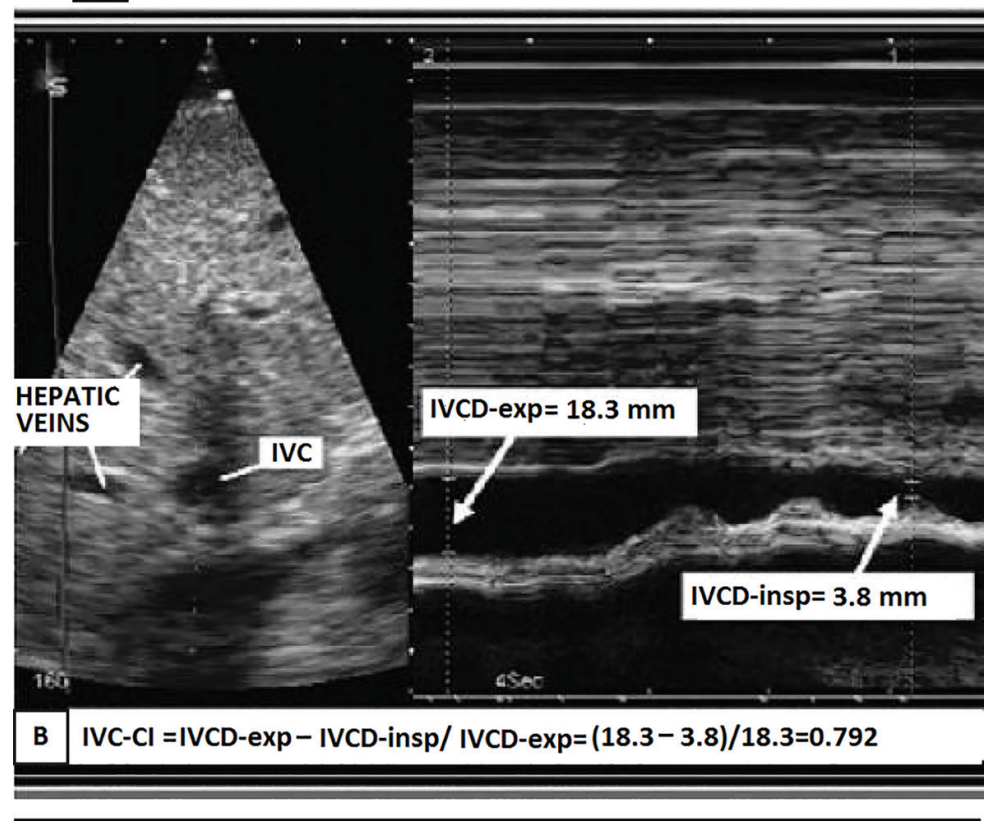

Figure 1. (A) Representation of the IVC collapsibility index (IVCCI) and (B) IVCCI measurement using M-mode ultrasonography. (A) IVCCl consists of the difference between the end-expiratory (IVCd-exp) and end-inspiratory (IVCd-insp) divided by IVCd-exp. (B) Based on the measurements in this example, the IVCCI would be $(18.3-3.8 \mathrm{~mm}) / 18.3 \mathrm{~mm}$, or $79.2 \%$. 
Table 3. Accuracy, Sensitivity and Specificity of Echocardiographic Measurements for Identification of RAP > $10 \mathrm{~mm}$ Hg According to Patel et al [21]

\begin{tabular}{|c|c|c|c|c|c|}
\hline & $\begin{array}{l}\text { 3D-RAVi } \geq \\
35 \mathrm{~mL} / \mathrm{m}^{2}+\mathrm{IVC} \geq 2 \mathrm{~cm}\end{array}$ & $\begin{array}{l}\text { 3D-RAVi } \geq \\
35 \mathrm{~mL} / \mathrm{m}^{2}\end{array}$ & $\begin{array}{l}3 D-R A V i \geq 35 \mathrm{~mL} / \mathrm{m}^{2}+ \\
I V C \geq 2 \mathrm{~cm}+\text { IVCCI }<40 \%\end{array}$ & $I V C \geq 2 \mathrm{~cm}$ & $\begin{array}{l}\text { IVC } \geq 2 \mathrm{~cm}+ \\
\text { IVCCI }<\mathbf{4 0 \%}\end{array}$ \\
\hline Accuracy & $0.88^{*}$ & 0.85 & 0.70 & 0.83 & 0.68 \\
\hline Sensitivity & $0.86 \dagger$ & 0.89 & 0.57 & 0.89 & 0.60 \\
\hline Specificity & 0.92 & 0.75 & 1.00 & 0.67 & 0.83 \\
\hline
\end{tabular}

Comparison of 3D-RAVi $\geq 35 \mathrm{~mL} / \mathrm{m}^{2}+$ IVC $\geq 2 \mathrm{~cm}$ versus IVC parameters alone. ${ }^{*} \mathrm{P}=0.038$ versus IVC $\geq 2 \mathrm{~cm}+I V C C I<40 \% ; \dagger P=0.041 \mathrm{vs}$. IVC $\geq 2 \mathrm{~cm}+\mathrm{IVCCI}<40 \%$. RAP: right atrial pressure; 3D-RAVi: three-dimensional right atrial volume index; IVC: inferior vena cava expiratory diameter; IVCCI: inferior vena cava collapsibility index.

sumed to indicate normal respiro-phasic motility of the IVC wall and would tend to exclude the possibility of hemodynamic congestion when combined with maximum values of IVC diameter $\leq 21 \mathrm{~mm}$ (Table 2).

In contrast, IVCCI values of $<50 \%$ would indicate increased likelihood of high RAP $(>8 \mathrm{~mm} \mathrm{Hg})$ and, if associated with maximum IVC diameter $>21 \mathrm{~mm}$, systemic venous congestion. The interpretation of less obviously pathognomonic conditions, such as cases where a maximum IVC diameter $\leq$ $21 \mathrm{~mm}$ is coupled with an IVCCI $<50 \%$, and cases where maximum IVC diameter $>21 \mathrm{~mm}$ is coupled with an IVCCI $\geq 50 \%$, is less clear; the ASE guidelines suggest categorizing patients as having normal RAP if secondary indicators of high RAP (Table 2) are absent and as having high RAP if the secondary indicators are present.

\section{Non-Invasive Diagnosis of High RAP According to Intensivists and Critical Care Physicians}

In overt contrast to the ASE guidelines, Stawicki et al [5] attribute reliable diagnostic value exclusively to the IVCCI; moreover, they distinguish only two categories for diagnostic purposes: values between 0 and $20 \%$, which are assumed to indicate pathological hypo-motility of the IVC wall and therefore a high $(>8 \mathrm{mmHg})$ RAP with high probability of right ventricular failure and values above $60 \%$, which would indicate normal or increased respiro-phasic IVC collapsibility, thereby excluding the presence of right heart failure (although not excluding, in the case of very high values, i.e., $90-100 \%$, the possibility of intravascular volume depletion).

With respect to their denial that simple measurement of maximum IVC diameter has reliable diagnostic value, Stawicki and colleagues are in fact in agreement with other authors [1, 4], i.e., they consider that any judgment based exclusively or predominantly on the maximum IVC diameter is subject to a large margin of error, especially given that in all cases of longstanding heart failure, the IVC outflow tract tends to become a flared and inelastic orifice at the entrance to the right atrium. Therefore, persistent hemodynamic congestion may be diagnosed erroneously in patients, in whom the IVC expiratory diameter remains high due to inelastic deformation of the ostium even when a normal RAP has been achieved as a consequence of adequate pharmacological therapy for acute cardiac decompensation. So, using maximum (expiratory) IVC diameter as the sole criterion for judging hemodynamic congestion would result in a high number of false positives, with the risk of an erroneous, excessive prolongation of intravenous diuretic therapy which has the potential to produce renal hypoperfusion and low cardiac output syndrome, both of which are related to overzealous unloading therapy.

\section{Non-Invasive Diagnosis of High RAP Based on the Combined Use of Three-Dimensional (3D) Echocardiography and Two-Dimensional (2D) Echocardiographic IVC Indices}

Patel et al [21] argued that in patients with heart failure, elevated RAP can be diagnosed highly accurately from the combination of 3D right atrial volume indexed to body surface area (3D-RAVi) plus IVC parameters. The study by Patel et al was the first to test the combination of 2D ultrasonographic indices such as IVC parameters with a 3D echo-derived parameter (3D-RAVi) as a non-invasive method of estimating RAP, using RAP values determined by catheterization of the right heart for reference. They explored the predictive value of several combinations of parameters with respect to the outcome variable "high RAP ": 1) 3D-RAVi $\geq 35 \mathrm{~mL} / \mathrm{m}^{2}$ in conjunction with maximum IVC diameter $\geq 2 \mathrm{~cm}$ (indicative of an RAP $>10$ $\mathrm{mm} \mathrm{Hg}$ ); 2) 3D-RAVi $\geq 3 \overline{5} \mathrm{~mL} / \mathrm{m}^{2}$; and 3) 3D-RAVi $\geq 35 \mathrm{~mL} /$ $\mathrm{m}^{2}$ combined with maximum IVC diameter $\geq 2 \mathrm{~cm}$ and IVCCI $<40 \%$ (indicative of RAP $>10 \mathrm{~mm} \mathrm{Hg}$ ). Traditional 2D IVC criteria were also included in the comparison, namely maximum IVC diameter $\geq 2 \mathrm{~cm}$ in isolation and maximum IVC diameter $\geq 2 \mathrm{~cm}$ alongside an IVCCI $<40 \%$.

The accuracy, sensitivity and specificity of these parameters for detection of RAP $>10 \mathrm{~mm} \mathrm{Hg}$ are shown in Table 3. Of the abovementioned parameters, the combination of RAVi $\geq 35 \mathrm{~mL} / \mathrm{m}^{2}$ plus dilated IVC (maximum IVC diameter $\geq 2$ $\mathrm{cm}$ ) yielded the optimal balance between sensitivity and specificity, i.e., the best diagnostic accuracy.

In contrast to Stawicki et al who argued that IVC ostium diameter alone should not be used as predictor of high RAP, Patel et al found that the combination of ostial diameter $\geq 2$ $\mathrm{cm}$ plus a $3 \mathrm{D}-\mathrm{RAVi} \geq 35 \mathrm{~mL} / \mathrm{m}^{2}$ yielded better diagnostic performance than the association of the first two parameters plus an IVCCI value of $<40 \%$. Thus, according to Patel et al, the diameter of the caval ostium could be assumed as a predictor of elevated RAP even without taking IVCCI into account; in 


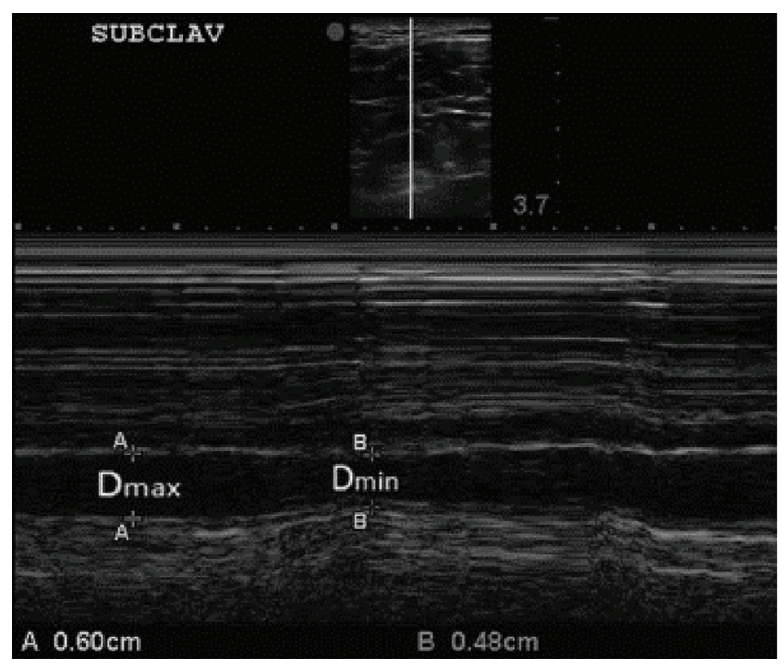

Figure 2. B-mode and M-mode views of the subclavian vein (SCV) with expiratory and inspiratory diameters measured. The clavicle is visualized anterior (above) the subclavian artery at the left side of the top image (superior to the SVC). Again, consistent with our approach to the IVCCI, we utilized minimal (inspiratory) and maximal (expiratory) diameters of the SCV. For further explanations, please see the text.

other words, IVC inspiratory collapse would be a less reliable parameter compared to the ostial diameter for identifying a state of elevated RAP.

The attempt by Patel et al to integrate the information provided by the $3 \mathrm{D}$ echocardiography with classic sonographic IVC indices for estimating RAP did not exert an effective impact on the diagnostic practice. Indeed, there was no change in ASE guidelines after publication of the study (in 2011), and $3 \mathrm{D}$ echocardiography did not receive validation as an additional assessment tool to be used in combination with IVC ultrasound as a non-invasive method of detecting elevated RAP.

\section{Further Attempts to Use Other Large Veins to Obtain an Indirect Estimate of RAP}

A non-invasive method of estimating RAP has long been an important goal for intensivists and critical care physicians and this has prompted some to search for alternative methods of estimating RAP, based either on ultrasonographic indices of great venous vessels or measurements of right atrial volume acquired using 3D echocardiography. Among the most interesting attempts to improve or diversify non-invasive methods for estimating RAP, those of Kent et al are to be mentioned [22, 23] (Fig. 2). They tried to study the correlation and the degree of concordance with the IVC inspiratory collapse exhibited by the similar inspiratory collapsibility of some major tributary veins of the venous caval system, by exploiting their easier accessibility for the echocardiographic probe due to their more superficial anatomic course. In fact Kent et al explored the respective indices of respiro-phasic collapsibility of the subclavian veins [22] as well as of internal jugular veins and femoral veins [23]. The selected methods for the study of the diagnostic validity of these parameters were the linear regression analysis and the Bland-Altman analysis for comparison of diagnostic techniques. These investigations revealed a poor correlation of the IVC sonographic findings with those obtained from the internal jugular veins and femoral veins [23], while a good correlation was documented between the degree of inspiratory collapse of the subclavian veins and that exhibited by IVC [22]. Linear regression analysis of paired measurements demonstrated a good correlation between the collapsibility of the subclavian veins and the IVC over a broad range of venous collapsibilities $(r=0.78)$. Moreover, Bland and Altman bias analysis demonstrated a mean collapsibility measurement bias of $-3.21 \%$ with a median collapsibility bias of $-1.67 \%$, indicating that values of the subclavian collapsibility index were generally slightly greater than IVCCI values [22].

\section{Conclusions}

There continues to be great interest in non-invasive methods of determining RAP among researchers committed to identifying the best techniques for evaluating circulating blood volume and diagnosing hypo- and hypervolemia. The concept of hypervolemia is a relative one; indeed, the term hypervolemia is used to refer to a state, in which there is relative hemodynamic overload of the circulatory system, which may precipitate cardiac insufficiency, i.e., the inability of the ventricular myocardium to mobilize sufficient intravascular blood for the nutritional and metabolic needs of the various organs and tissues. An increase in right atrial volume and dilation and respiro-phasic hypo-motility of the IVC distal tract have been identified as reliable indicators of hemodynamic congestion. The latter indicators have also been shown to be good markers of cardiac hemodynamic overload and potential or current heart failure. The various criteria for elevated RAP are based either on morphofunctional evaluation of the distal segment of IVC or measurements of right atrial volume indexed to body surface area taken using 3D echocardiographic technique, or both. Validation of these criteria is based on the grade of correlation with the measurements taken from the right heart catheterization, which has been proven to be quite satisfactory. Further advances in non-invasive methods of estimating RAP are expected, especially because 3D echocardiography offers now the possibility of estimating right atrial volume more precisely than previously. Moreover, further research is warranted, to establish whether the correlation between mean RAP and right atrial volume is reliable and constant across the various phases of the cardiac cycle.

\section{References}

1. Moreno FL, Hagan AD, Holmen JR, Pryor TA, Strickland $\mathrm{RD}$, Castle CH. Evaluation of size and dynamics of the inferior vena cava as an index of right-sided cardiac function. Am J Cardiol. 1984;53(4):579-585.

2. Kircher BJ, Himelman RB, Schiller NB. Noninvasive estimation of right atrial pressure from the inspiratory collapse of the inferior vena cava. Am J Cardiol. 1990;66(4):493-496. 
3. Brennan JM, Blair JE, Goonewardena S, Ronan A, Shah D, Vasaiwala S, Kirkpatrick JN, et al. Reappraisal of the use of inferior vena cava for estimating right atrial pressure. J Am Soc Echocardiogr. 2007;20(7):857-861.

4. Blehar DJ, Dickman E, Gaspari R. Identification of congestive heart failure via respiratory variation of inferior vena cava diameter. Am J Emerg Med. 2009;27(1):71-75.

5. Stawicki SP, Braslow BM, Panebianco NL, Kirkpatrick JN, Gracias VH, Hayden GE, Dean AJ. Intensivist use of hand-carried ultrasonography to measure IVC collapsibility in estimating intravascular volume status: correlations with CVP. J Am Coll Surg. 2009;209(1):55-61.

6. Richard C, Monnet X, Teboul JL. Pulmonary artery catheter monitoring in 2011. Curr Opin Crit Care. 2011;17(3):296-302.

7. Swan HJ, Ganz W, Forrester J, Marcus H, Diamond G, Chonette D. Catheterization of the heart in man with use of a flow-directed balloon-tipped catheter. N Engl J Med. 1970;283(9):447-451.

8. Kuppahally SS, Michaels AD, Tandar A, Gilbert EM, Litwin SE, Bader FM. Can echocardiographic evaluation of cardiopulmonary hemodynamics decrease right heart catheterizations in end-stage heart failure patients awaiting transplantation? Am J Cardiol. 2010;106(11):16571662.

9. Taleb M, Khuder S, Tinkel J, Khouri SJ. The diagnostic accuracy of Doppler echocardiography in assessment of pulmonary artery systolic pressure: a meta-analysis. Echocardiography. 2013;30(3):258-265.

10. Chang PP, Longenecker JC, Wang NY, Baughman KL, Conte JV, Hare JM, Kasper EK. Mild vs severe pulmonary hypertension before heart transplantation: different effects on posttransplantation pulmonary hypertension and mortality. J Heart Lung Transplant. 2005;24(8):9981007.

11. D'Alto M, Alfano D, Maiello C, Sarubbi B, Santoro G, Argiento P, Galdieri N, et al. Complex multidrug therapy in a patient with pulmonary hypertension before and after orthotopic heart transplantation. A case report. J Cardiovasc Med (Hagerstown). 2007;8(11):950-952.

12. Cioffi G, Tarantini L, Pulignano G, Del Sindaco D, De Feo S, Opasich C, Dilenarda A, et al. Prevalence, predictors and prognostic value of acute impairment in renal function during intensive unloading therapy in a community population hospitalized for decompensated heart failure. J Cardiovasc Med (Hagerstown). 2007;8(6):419-427.

13. Goonewardena SN, Gemignani A, Ronan A, Vasaiwala S, Blair J, Brennan JM, Shah DP, et al. Comparison of hand-carried ultrasound assessment of the inferior vena cava and $\mathrm{N}$-terminal pro-brain natriuretic peptide for predicting readmission after hospitalization for acute decompensated heart failure. JACC Cardiovasc Imaging. 2008;1(5):595-601.

14. Gheorghiade M, Follath F, Ponikowski P, Barsuk JH,
Blair JE, Cleland JG, Dickstein K, et al. Assessing and grading congestion in acute heart failure: a scientific statement from the acute heart failure committee of the heart failure association of the European Society of Cardiology and endorsed by the European Society of Intensive Care Medicine. Eur J Heart Fail. 2010;12(5):423-433.

15. Nohria A, Hasselblad V, Stebbins A, Pauly DF, Fonarow GC, Shah M, Yancy CW, et al. Cardiorenal interactions: insights from the ESCAPE trial. J Am Coll Cardiol. 2008;51(13):1268-1274.

16. De Vecchis R, Baldi C. Inferior Vena Cava and Hemodynamic Congestion. Res Cardiovasc Med. 2015;4(3):e28913.

17. De Vecchis R, Ciccarelli A, Ariano C. Inferior Vena Cava collapsibility and heart failure signs and symptoms: new insights about possible links. Arq Bras Cardiol. 2012;98(6):544-552.

18. De Vecchis R, Ariano C, Fusco A, Ciccarelli A, Cioppa C, Giasi A, Esposito C, et al. Ultrasound evaluation of the inferior vena cava collapsibility index in congestive heart failure patients treated with intravenous diuretics: new insights about its relationship with renal function: an observational study. Anadolu Kardiyol Derg. 2012;12(5):391400.

19. Rudski LG, Lai WW, Afilalo J, Hua L, Handschumacher MD, Chandrasekaran K, Solomon SD, et al. Guidelines for the echocardiographic assessment of the right heart in adults: a report from the American Society of Echocardiography endorsed by the European Association of Echocardiography, a registered branch of the European Society of Cardiology, and the Canadian Society of Echocardiography. J Am Soc Echocardiogr. 2010;23(7):685-713; quiz 786-688.

20. Porter TR, Shillcutt SK, Adams MS, Desjardins G, Glas KE, Olson JJ, Troughton RW. Guidelines for the use of echocardiography as a monitor for therapeutic intervention in adults: a report from the American Society of Echocardiography. J Am Soc Echocardiogr. 2015;28(1):40-56.

21. Patel AR, Alsheikh-Ali AA, Mukherjee J, Evangelista A, Quraini D, Ordway LJ, Kuvin JT, et al. 3D echocardiography to evaluate right atrial pressure in acutely decompensated heart failure correlation with invasive hemodynamics. JACC Cardiovasc Imaging. 2011;4(9):938-945.

22. Kent A, Bahner DP, Boulger CT, Eiferman DS, Adkins EJ, Evans DC, Springer AN, et al. Sonographic evaluation of intravascular volume status in the surgical intensive care unit: a prospective comparison of subclavian vein and inferior vena cava collapsibility index. J Surg Res. 2013;184(1):561-566.

23. Kent A, Patil P, Davila V, Bailey JK, Jones C, Evans DC, Boulger CT, et al. Sonographic evaluation of intravascular volume status: Can internal jugular or femoral vein collapsibility be used in the absence of IVC visualization? Ann Thorac Med. 2015;10(1):44-49. 